EXTENDED REPORT

\title{
Is visual outcome compromised when next day review is omitted after phacoemulsification surgery? A randomised control trial
}

\author{
C G Tinley, A Frost, K N Hakin, W McDermott, P Ewings
}

Br J Ophthalmol 2003;87:1350-1355

See end of article for authors' affiliations

Correspondence to Mr Christopher G Tinley, Southampton University Hospitals Trust Southampton, UK cgtinley@doctor.com

Accepted for publication 31 March 2003
Aims: To examine the safety implications of omitting first day clinical review following phacoemulsification cataract surgery.

Methods: 362 patients were randomly assigned to "same day discharge" (SDD) or "next day review" (NDR). All patients were reviewed approximately 2 weeks after surgery.

Results: Of the 174 patients randomised to NDR, 14 (8.0\%) were treated for raised intraocular pressure $(25-48 \mathrm{~mm} \mathrm{Hg})$ on the first postoperative day. Four received increased topical steroids for uveitis (two) and corneal oedema (two). One patient was treated for a significant wound leak. 12 (6.9\%) required additional reviews before 2 week follow up for treatment of the following complications: drop toxicity (six), raised intraocular pressure (five), and corneal abrasion (one). Of the 188 randomised to SDD, six (3.2\%) returned to the department before the planned review for reassurance of patients' concerns regarding eye symptoms (three), drop toxicity (one) and follow up of previously raised intraocular pressure (one). There were two cases of iris prolapse in the SDD group. In one case, the complication was anticipated and early review had been arranged. Postoperative acuities of $6 / 12$ or better were achieved in $83 \%$ of both SDD and NDR patients ( $p=0.96$ by $\chi^{2}$ test). Postoperative quality of life scores at 4 months indicating "no or hardly any concern about vision" (VCM1 questionnaire index $<1.0$ ) were achieved in 67\% SDD and $72.5 \%$ NDR $(p=0.26)$.

Conclusion: The intention to discharge patients on the day of surgery, with planned postoperative review at 2 weeks, was associated with a low frequency of serious ocular complications. Differences in the proportions achieving a good visual outcome between the two groups, based on 2 week visual acuity and 4 month quality of life, were not significant.
A recent UK government directive ${ }^{1}$ to increase cataract surgery rates (to 250000 per annum by 2003) has resulted in a growing trend towards discharging patients on the day of surgery, without first day postoperative review. Besides considerably reducing service costs, many patients prefer not to have to return the following day. Traditional first day postoperative review, however, has several advantages: the detection of complications, collection of data for audit purposes, reassurance for the patient, and training of staff.

Previous non-randomised studies ${ }^{2-8}$ have investigated the safety of discharging patients on the day of surgery, with review deferred until the first routine outpatient follow up visit, usually $1-2$ weeks after surgery. Conclusions have been difficult to draw because of a lack of direct comparisons between patient groups receiving alternative forms of care. In one study, ${ }^{5}$ where such a comparison was made, no additional risk was detected, but the patients were not randomly allocated.

The purpose of this randomised control trial was to detect differences in clinical outcomes, if present, between patients who were randomised either to be reviewed the day after surgery ("next day review," NDR), or to have the first day review omitted ("same day discharge," SDD). The outcomes measured were frequency of postoperative complications, visual acuity, and vision related quality of life.

\section{METHODS}

The study protocol was approved by the local research ethics committee and was conducted in accordance with the Declaration of Helsinki.

\section{Sampling and recruitment}

The target study population comprised all patients scheduled for elective cataract surgery at Musgrove Park Hospital, Taunton, a district general hospital in the largely rural county of Somerset, UK, between 26 October 2000 and 18 April 2001.

The exclusion criteria were as follows: inadequate social support for overnight care at home, severely limited visual potential in the fellow eye, severe systemic disease limiting activity (ASA grade III or worse), patient undergoing another simultaneous ophthalmic procedure, patient with learning disability or dementia, and age less than 16 years.

Study information and preoperative questionnaires were mailed to patients on the waiting list together with the preoperative assessment clinic (POAC) appointment dates. The questionnaires were self completed at home and returned to the POAC nurse on the date of the assessment. The patient signed a study consent form if he/she was eligible for the study and was willing to participate.

\section{Random assignment}

It was anticipated that previous experience of surgery might influence the questionnaire responses. Randomisation was therefore stratified according to whether patients were having their first or second cataract extraction. Two separate, block randomised allocation lists were generated by computer. One list was for first eye cataract operations and the other for second eye operations. The separate allocations (SDD or NDR) were then sealed inside opaque envelopes before delivery to the POAC. The contents were unknown to all staff and investigators who dealt with patients. The sealed envelopes bore on the outside only first or second eye and a 
trial identification number. After the patient was enrolled in the trial, a consecutive envelope was opened from the appropriate set. Once the envelope was opened, the patients, care providers, and investigators were no longer masked to the allocation.

\section{SDD care pathway}

Patients assigned to SDD had their eye pads removed 23 hours after surgery. The eyes were cleaned and inspected with a torch by a nurse on the ward. Discharge information was given to patients, which included a contact telephone number for emergencies. If the nurses were satisfied with a torch inspection of the eye, the patients were discharged and a 2 week outpatient clinic follow up appointment was made. The operating surgeon could change the postoperative care plan for clinical reasons if he/she believed it was necessary to do so.

\section{NDR care pathway}

Patients allocated to "next day review" could either return home for the first postoperative night, or opt to stay in hospital in a low dependency "hostel" bed without nursing care. This system was arranged to accommodate patients who would have difficulties returning early the next day because of problems with transport. The operated eyes were left with pads on overnight. A doctor examined the patients the following morning, after which, if satisfactory, they were discharged and a 2 week outpatient clinic follow up appointment made.

\section{Principal outcomes}

Complications within the first month and visual acuities at the 2 week follow up visit were assessed by review of the case notes. Acuity was assessed as the best (unaided or pinhole) completed Snellen lines. Vision related quality of life impairment was measured using the VCMl questionnaire. ${ }^{9}$ The VCMl contains 10 broadly applicable items referring to physical, social, and psychological issues, and acts as a global measure of concern about vision. The VCMl score ranges from 0.0 (no concern) to 5.0 (extreme concern) and is strongly associated with responses to questions about a wide range of quality of life issues including mobility, reading, and leisure.

Postoperative questionnaires were mailed to patients 4 months after the date of surgery with stamped, return envelopes. Patients whose questionnaires were not returned within 2 weeks were sent another. If still not returned, the patient was interviewed by telephone. The reliability of postal and telephone administration has been tested. ${ }^{10}$

The acceptability of the two different care pathways was assessed in the post-operative questionnaire by asking, "If you had to have the same cataract operation again, would you prefer to be discharged on the same day as the operation, or would you prefer to return the next day to have your eye checked?"

\section{Statistical methods}

Power calculation was based on an estimate of the likely postoperative VCMl questionnaire scores. The VCMl score standard deviation, calculated in 70 postoperative cataract patients from a random population sample, was approximately 1.0. A mean difference in questionnaire scores of 0.3 between the two groups was deemed to be of clinical importance. The power to detect such a difference was 0.8 (two tailed) with 186 patients per group.

The outcomes were analysed by group of initial allocation, rather than final management. The Mann-Whitney test was used to compare 2 week visual acuities and 4 month VCMI scores between the two groups. The $\chi^{2}$ test was used to compare the proportion of patients achieving a VCMl score of less than 1.0, indicating "no or hardly any concern about vision". The $\chi^{2}$ test was also used to compare the proportion of patients achieving 6/12 or better visual acuity. Logistic regression analysis provided clinical predictors for a good visual outcome.

\section{RESULTS}

\section{Representativeness}

Table 1 demonstrates that the age and sex of the patients included in the sample were similar to that of the national average (1997-8). ${ }^{11}{ }^{12}$ Preoperative visual acuity was better in the sample group (2000-1), which may reflect the trend towards earlier surgery for mild to moderate cataracts. The National Cataract Surgery Survey ${ }^{11}$ listed only diabetic retinopathy, age-related macular degeneration, glaucoma, and amblyopia as co-morbid factors. Our trial recorded a larger range of co-existing pathologies, which may explain the greater percentage of patients in the trial with ocular comorbidity in comparison with the national average.

\section{Recruitment}

The recruitment of patients is described graphically in Figure 1. A total of 229 patients refused entry into the trial. The main reasons given for refusal were a preference for same day discharge $(40 \%$ of patients) and transport difficulties $(25 \%)$. The recruitment rate was $64 \%$; 38 were withdrawn during the trial. Of those withdrawn, 15 NDR patients decided on the day of surgery that they did not wish to return for follow up the following day and 13 patients' operations were cancelled or postponed. Four protocol violations included patients who were randomised to NDR, but who were not reviewed the next day. Four patients died shortly after inclusion into the trial and two sets of patient notes were missing. Of the 362 patients retained in the study, 188 were randomised to SDD and 174 to NDR; 71 (41\%) of NDR patients opted for an overnight hostel admission.

A total of approximately 1100 cataract patients went through the department during the 6 month recruitment period (26 October 2000-18 April 2001). Follow up ended on the 31 August 2001 and the median duration of follow up was 125 days (approximately 4 months).

\section{Surgery}

Pupils were dilated preoperatively with $10 \%$ phenylephrine and $1 \%$ cyclopentolate; $79 \%$ of the cataract operations were performed under peribulbar local anaesthesia; 18\% were under general anaesthesia and 3\% were topical. Consultants performed $27 \%$ of the operations, while intermediate grades, including staff grades, registrars, and fellows, performed the remainder. Clear corneal incisions were used and phacoemulsification was the technique of choice. Elective extracapsular cataract extractions were undertaken in two cases

Table 1 Comparison of patient demographics, preoperative visual acuity, and ocular co-morbidity between study population and national average

\begin{tabular}{lll}
\hline & Taunton & National average \\
\hline Mean age (years) & 75.4 & 76.3 \\
Age $>70$ years & $77 \%$ & $78 \%$ \\
Females & $62 \%$ & $65 \%$ \\
Preop visual acuity & & \\
$6 / 12$ or better & $65 \%$ & $27 \%$ \\
$6 / 18-6 / 60$ & $28 \%$ & $52 \%$ \\
Worse than 6/60 & $7 \%$ & $21 \%$ \\
Ocular co-morbidity & $36 \%$ & $28 \%$ \\
\hline
\end{tabular}




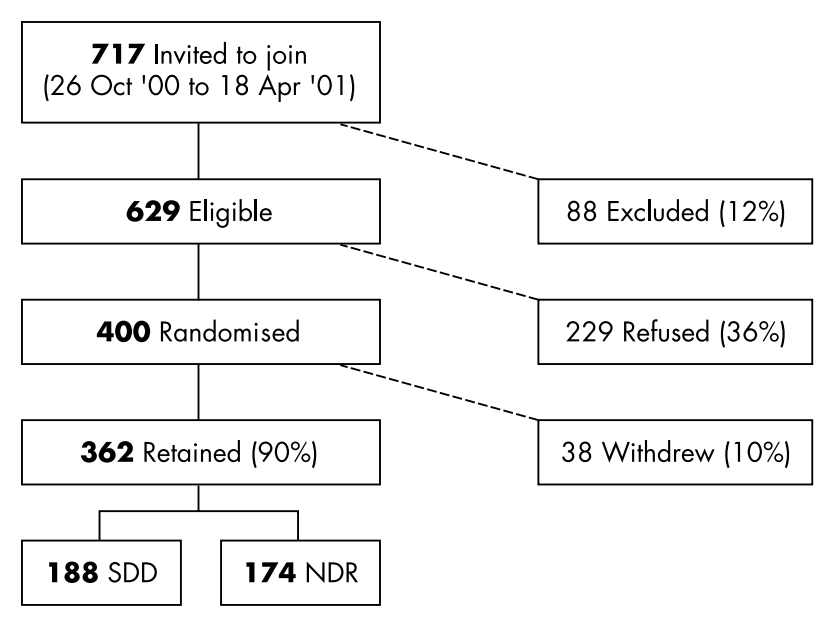

Figure 1 Recruitment diagram.

with dense nuclear cataracts. Healon was the intraocular viscoelastic agent most commonly used. In all cases injectable acrylic or silicone posterior chamber intraocular lenses were inserted, except in a single case of a dropped nucleus, in which no lens was inserted. Irrigation and aspiration of cortical remnants was performed either automated or manually. Surgical wounds were hydrated with balanced saline solution and $20 \%$ were sutured. Subconjunctival cefotaxime and betamethasone were used at completion of surgery. Prophylactic medication to lower the intraocular pressure (IOP) was not routinely administered. Postoperatively, a transparent plastic eye shield was provided for use at night. Maxitrol drops (dexamethasone, neomycin, and polymixin B) four times a day were prescribed.

\section{Crossovers}

Crossovers, in the context of this trial, are described as patients whose management differed from the initial management allocation-that is, an SDD patient who was subsequently managed as an NDR and vice versa. 13 SDD patients were reviewed on day l (because of operative or anaesthetic problems) and I NDR patient was discharged on the day of surgery.

\section{Baseline comparisons}

Table 2 shows that the two groups were well balanced in terms of sex, age, and proportion having first eye surgery. Percentages of patients having ocular co-pathology were similar between the two groups.

\section{Operative complications}

Operative complications (Table 3) were infrequent. The most common was posterior capsule rupture, three $(1.6 \%)$ in the SDD group and four $(2.3 \%)$ in the NDR group. None of the patients with intraoperative iris prolapse (six SDD, three NDR) had prolapse at NDR or at 2 week follow up.

Peroperative prophylactic IOP lowering medication was prescribed for seven patients in each of the two groups, usually following difficult surgery. Glaucoma patients

Table 2 Baseline comparison between the two groups

\begin{tabular}{|c|c|c|c|c|}
\hline & \multicolumn{2}{|l|}{ SDD } & \multicolumn{2}{|l|}{ NDR } \\
\hline & No & $\%$ & No & $\%$ \\
\hline Total & 188 & & 174 & \\
\hline Males & 67 & 36 & 70 & 40 \\
\hline Females & 121 & 64 & 104 & 60 \\
\hline Mean age & 76 & & 75 & \\
\hline Median age & 78 & & 75 & \\
\hline Age range & 43-95 & & 37-92 & \\
\hline First eye surgery & 107 & 57 & 103 & 59 \\
\hline Second eye surgery & 81 & 43 & 71 & 41 \\
\hline \multicolumn{5}{|l|}{ Anaesthetic } \\
\hline Topical & 7 & 4 & 3 & 2 \\
\hline Peribulbar & 149 & 79 & 136 & 78 \\
\hline General & 32 & 17 & 35 & 20 \\
\hline \multicolumn{5}{|l|}{ Surgeon } \\
\hline Consultant & 56 & 30 & 42 & 24 \\
\hline Middle grade & 132 & 70 & 130 & 75 \\
\hline Senior house officer & 0 & 0 & 2 & 1 \\
\hline \multicolumn{5}{|l|}{ Co-existing pathology } \\
\hline Total & 69 & 37 & 60 & 34 \\
\hline Macular pathology & 44 & 23 & 38 & 22 \\
\hline Glaucoma & 14 & 7 & 15 & 9 \\
\hline Ocular hypertension & 2 & 1 & 1 & 1 \\
\hline Optic neuropathy & 2 & 1 & 2 & 1 \\
\hline Amblyopia & 1 & 1 & 3 & 2 \\
\hline Uveitis & 3 & 2 & 4 & 2 \\
\hline Previous intraocular surgery & 11 & 6 & 15 & 9 \\
\hline Other & 11 & 6 & 6 & 3 \\
\hline \multicolumn{5}{|c|}{ Preoperative Snellen visual acuities } \\
\hline $6 / 12$ or better & 120 & 64 & 115 & 66 \\
\hline $6 / 18-6 / 60$ & 55 & 29 & 47 & 27 \\
\hline Worse than $6 / 60$ & 13 & 7 & 12 & 7 \\
\hline Median follow up (days) & 125 & & 125 & \\
\hline Range of follow up (days) & $57-311$ & & $62-276$ & \\
\hline \multicolumn{5}{|c|}{$\begin{array}{l}\text { Ocular pathology definitions: macular pathology ncludes diabetic maculopathy, age related macular } \\
\text { degeneration, epiretinal membrane, macular hole, myopic maculopathy; glaucoma: documented clinical } \\
\text { diagnosis; ocular hypertension: raised IOP without disc changes or field defect; optic neuropathy: optic nerve } \\
\text { damage resulting in loss of VA, field defect, altered pupil reflexes (RAPD) or colour vision impairment; amblyopia } \\
\text { as stated in patient notes; uveitis: previous documented episode of anterior uveitis; previous intraocular surgery: } \\
\text { includes laser trabeculoplasty, trabeculectomy, corneal graft, retinal detachment repair and vitrectomy; other: other } \\
\text { vision impairing conditions not defined above. }\end{array}$} \\
\hline
\end{tabular}


Table 3 Peroperative and postoperative complications

\begin{tabular}{|c|c|c|c|c|c|c|}
\hline \multirow[b]{2}{*}{ Peroperative complications } & \multicolumn{3}{|l|}{ SDD } & \multicolumn{3}{|l|}{ NDR } \\
\hline & No & \multicolumn{2}{|l|}{$\%$} & No & \multicolumn{2}{|l|}{$\%$} \\
\hline Perop IOP Rx & 7 & \multicolumn{2}{|l|}{4} & 7 & \multicolumn{2}{|l|}{4} \\
\hline Posterior capsule rupture & 3 & \multicolumn{2}{|l|}{2} & 4 & \multicolumn{2}{|l|}{2} \\
\hline Expulsive haemorrhage & 0 & \multicolumn{2}{|l|}{0} & 1 & \multicolumn{2}{|l|}{1} \\
\hline Iris prolapse & 6 & \multicolumn{2}{|l|}{3} & 3 & \multicolumn{2}{|l|}{2} \\
\hline Other & 1 & \multicolumn{2}{|l|}{1} & 3 & \multicolumn{2}{|l|}{2} \\
\hline Total & 17 & \multirow{2}{*}{\multicolumn{2}{|c|}{9}} & 18 & \multicolumn{2}{|l|}{10} \\
\hline \multicolumn{5}{|l|}{ Interventions at NDR } & & \\
\hline IOP Rx (25-48 mm Hg) & & & & 14 & \multicolumn{2}{|l|}{8} \\
\hline Increased steroids & & & & 4 & \multicolumn{2}{|l|}{2} \\
\hline Wound leak & & & & 1 & \multicolumn{2}{|l|}{1} \\
\hline Loose suture & & & & 1 & \multicolumn{2}{|l|}{1} \\
\hline Punctate epitheliopathy & & & & 1 & \multicolumn{2}{|l|}{1} \\
\hline IOL subluxation & & & & 0 & \multicolumn{2}{|l|}{0} \\
\hline Iris prolapse & & & & 0 & \multicolumn{2}{|l|}{0} \\
\hline Total & & & & 21 & \multicolumn{2}{|l|}{12} \\
\hline \multicolumn{7}{|l|}{ Postoperative complications } \\
\hline Drop toxicity & 1 & 1 & 10 & 6 & 3 & $9,9,9,10,11,14$ \\
\hline IOP Rx & 1 & 1 & 1 & 5 & 3 & $0,2,2,2,3,14$ \\
\hline Corneal abrasion & 0 & 0 & & 1 & 1 & \\
\hline Patient reassurance & 3 & 2 & $0,2,3$ & 0 & 0 & \\
\hline Iris prolapse & 1 & 1 & 3 & 0 & 0 & \\
\hline Total & 6 & 3 & & 12 & 7 & \\
\hline \multicolumn{7}{|l|}{ Postoperative complications } \\
\hline Iris prolapse & 1 & 1 & & 0 & 0 & \\
\hline Uveitis & 2 & 1 & & 2 & 1 & \\
\hline Cystoid macular oedema & 1 & 1 & & 0 & 0 & \\
\hline Drop toxicity & 6 & 3 & & 3 & 2 & \\
\hline Tight corneal suture & 0 & 0 & & 1 & 1 & \\
\hline Total & 10 & 5 & & 6 & 3 & \\
\hline \multicolumn{7}{|c|}{$\begin{array}{l}\text { Postoperative complication definitions: IOP Rx: intraocular pressure treatment; increased IOP: sufficient to alter } \\
\text { standard management of Maxitrol drops four times a day; uveitis: anterior uveitis sufficient to increase dose or } \\
\text { prolong course of topical steroid; wound leak: sufficient to justify padding, bandage contact lens, re-suturing, re- } \\
\text { admission or delayed discharge; dislocated/subluxed IOL: malposition of intraocular lens as documented in notes } \\
\text { or incorrect placement of lens; iris prolapse: protrusion of iris through or into corneal section; cystoid macular } \\
\text { oedema: clinical diagnosis documented in the notes; drop toxicity: increasing patient discomfort immediately after } \\
\text { instillation of drops or diffuse corneal epitheliopathy; other: any postoperative complication not defined above. }\end{array}$} \\
\hline
\end{tabular}

were not routinely prescribed prophylactic IOP lowering medication.

\section{Postoperative complications}

Of the patients randomised to next day review, $12 \%$ received alterations in standard management on day 1 for reasons listed in Table 3. Fourteen patients were prescribed oral acetazolamide for raised IOP and four patients' topical steroid dosage was increased for anterior uveitis (two) and corneal oedema (two). A bandage contact lens was inserted for one wound leak and the patient with marked punctate epitheliopathy was prescribed preservative free lubricants.

Reasons for patients' return to the eye unit before the 2 week follow up visit are also found in Table 3. One SDD patient, who underwent complicated surgery (posterior capsule rupture), was reviewed the next day and was found to have a raised IOP $(28 \mathrm{~mm} \mathrm{Hg})$. Oral acetazolamide was prescribed and further review planned within 48 hours. An

Table 4 Postoperative Snellen visual acuities

\begin{tabular}{|c|c|c|c|c|}
\hline Postop VA & SDD & $\%$ & NDR & $\%$ \\
\hline $6 / 4$ & 1 & 0.5 & 1 & 1 \\
\hline $6 / 5$ & 4 & 2 & 5 & 3 \\
\hline $6 / 6$ & 26 & 14 & 28 & 16 \\
\hline $6 / 9$ & 97 & 52 & 91 & 52 \\
\hline $6 / 12$ & 28 & 15 & 19 & 11 \\
\hline $6 / 18$ & 19 & 10 & 12 & 7 \\
\hline $6 / 24$ & 1 & 0.5 & 6 & 3 \\
\hline $6 / 36$ & 3 & 1.5 & 3 & 2 \\
\hline $6 / 60$ & 3 & 1.5 & 3 & 2 \\
\hline $\mathrm{CF}$ & 6 & 3 & 4 & 2 \\
\hline $\mathrm{HM}$ & 0 & 0 & 2 & 1 \\
\hline PL & 0 & 0 & 0 & 0 \\
\hline NPL & 0 & 0 & 0 & 0 \\
\hline Total & 188 & 100 & 174 & 100 \\
\hline
\end{tabular}




\begin{tabular}{|c|c|c|c|c|}
\hline \multirow[b]{2}{*}{ VCMI score } & \multicolumn{2}{|l|}{ SDD } & \multicolumn{2}{|l|}{ NDR } \\
\hline & No & $\%$ & No & $\%$ \\
\hline 0.0 & 45 & 24 & 54 & 31 \\
\hline $0.1-1.0$ & 88 & 48 & 74 & 43 \\
\hline $1.1-2.0$ & 34 & 18 & 22 & 13 \\
\hline $2.1-3.0$ & 11 & 6 & 14 & 8 \\
\hline $3.1-4.0$ & 4 & 2 & 6 & 4 \\
\hline $4.1-5.0$ & 3 & 2 & 1 & 1 \\
\hline $\begin{array}{l}\text { Total } \\
\text { Questionnaire administration }\end{array}$ & 185 & 100 & 171 & 100 \\
\hline Postal & 163 & 88 & 156 & 91 \\
\hline Telephone & 22 & 12 & 15 & 9 \\
\hline Opticians eye test since surgery (self reported) & 3 & 1 & 3 & 2 \\
\hline Missing VCMIs* & 136 & 72 & 124 & 71 \\
\hline
\end{tabular}

iris prolapse, not present on day 1 , was noted in this patient on day 3, which required surgical repositioning. The prolapse occurred despite the wound having been sutured.

At the 2 week follow up visit, one case of iris prolapse was detected in an SDD patient. The patient, whose postoperative visual acuity was 6/9, did not require further surgery and was discharged after a second follow up visit at 1 month.

\section{Visual acuity}

Two week visual acuities are given in Table 4. Statistical comparison showed no significant difference in acuities between the two groups ( $\mathrm{p}=0.96$ with the Mann-Whitney test); $6 / 12$ vision or better was achieved in $83 \%$ of both SDD and NDR patients $\left(p=0.96 \chi^{2}\right.$; relative risk $0.99 ; 95 \%$ CI $0.63-1.55)$.

\section{Vision related quality of life}

Four month postoperative vision related quality of life scores are shown in Table 5 . There was no statistically significant difference in the scores between the two groups $(p=0.29$ Mann-Whitney). Scores of $<1.0$, which indicate "no or hardly any concern about vision," were achieved in $67 \%$ of SDD patients and $72.5 \%$ of NDR patients $\left(\mathrm{p}=0.26 \chi^{2}\right.$; relative risk $=1.20 ; 95 \%$ confidence interval $0.87-1.65)$.

\section{Delayed diagnoses due to SDD}

One iris prolapse was first diagnosed at 2 week follow up. The complication did not result in a compromised visual outcome, however, and did not require further surgical intervention. The second case of iris prolapse occurred in a sutured wound in an SDD crossover and was diagnosed on day 3. Notably, the iris was not prolapsed at day 1 review.

\section{Number of visits in each group}

In the NDR group a larger number of additional visits were made to the department before 2 week follow up (12 NDR $v$ six SDD). In both groups, the extra visits were distributed throughout the 2 week period (Table 3 ).

Within the first month of surgery, the 188 SDD patients made total of 227 visits to the department ( 1.2 per patient).

\begin{tabular}{llll}
\hline \multicolumn{2}{l}{ Table 6} & \multicolumn{2}{l}{ Patient management } \\
\hline Managed & Prefer SDD(\%) & Prefer NDR(\%) & Unsure(\%) \\
\hline SDD, first eye & 69 & 19 & 12 \\
SDD, second eye & 64 & 25 & 11 \\
NDR, first eye & 24 & 69 & 7 \\
NDR, second eye & 30 & 59 & 11 \\
\hline
\end{tabular}

The 174 NDR patients made 375 visits in first month (2.2 per patient). This translates to 1.0 visit saved for every patient managed as a "same day discharge."

\section{Acceptability of SDD}

Of the 229 patients who were eligible to enter the trial but refused, $147(64 \%)$ did not want to return or would have difficulty returning the next day. A further 15 NDR patients decided on the day of surgery not to return the following day for review.

Of those who continued to participate in the study, patients' responses seem to be influenced by how they were managed. Most stated a preference for the management they had received. (Table 6) Patients in the NDR group having their second cataract operations had a slightly larger proportion favouring SDD as opposed to patients in the same group undergoing their first operation. This could be because they had been managed as an SDD during their previous cataract surgery before this study.

\section{DISCUSSION}

Reported frequencies of complications presenting on day 1 vary between $3 \%{ }^{4}$ and $41 \%{ }^{6}$ Direct comparisons cannot be made, however, as some studies excluded patients with coexisting ocular pathology and most of the larger studies were conducted retrospectively. ${ }^{3-8}$ As is the case in this trial, the main problem identified at first day review was a raised intraocular pressure. The literature is divided as to the importance of detecting this postoperative pressure rise and much has been speculated regarding its appropriate management.

It is well known that the acute postoperative pressure rise after phacoemulsification is usually transient, with a peak occurring between 3 and 6 hours after surgery. ${ }^{13}{ }^{14}$ It has also been shown that this pressure pattern may be more common in glaucoma patients. ${ }^{15} 16$ However, the risk of visual field deterioration or other vascular events during this period is uncertain. As regards the role of first day review, it can be argued that a policy of treating an elevated IOP that persists at 24 hours may only marginally reduce the potential risks, because the peak pressure is likely to have occurred earlier. For clinicians concerned about the possible harmful effects of the pressure spike, recent studies ${ }^{17-19}$ suggest that prophylactic topical IOP lowering agents may be effective in preventing or reducing it.

The National Cataract Surgery Survey ${ }^{11}$ reported corneal oedema to be the most common postoperative complication within the first 48 hours. It is questionable whether this can be viewed as a separate entity requiring treatment. Defining 
it as a complication may influence doctors to treat the condition, perhaps unnecessarily.

All postoperative patients have a degree of anterior uveitis on day 1. The majority of patients at day 1 have not yet commenced topical anti-inflammatory medication. The severity of uveitis deemed necessary to treat is subjective and therefore in some cases excessive treatment may be of no clinical benefit.

Iris prolapse and other complications such as endophthalmitis and retinal detachment are rare and are not necessarily detected on the first postoperative day. This is demonstrated in our study, where one of the two cases of iris prolapse occurred in a sutured wound after the first day review. No cases of endophthalmitis or retinal detachment were reported in any of the studies looking at complications on day 1 , which comprised a total of more than 2000 patients.

\section{Acceptability of SDD}

A surprisingly large proportion of patients eligible for the trial (36\%) refused entry. Most of these patients quoted the inconvenience of returning the next day as the determining factor. Another closely related factor cited was transport difficulties. Many patients would have had to travel considerable distances from outlying villages, early in the day. It is self evident that patients will not want to make an unnecessary extra visit to the hospital. Postoperative preferences (Table 6), however, indicate that the majority of patients in both groups were satisfied with the care pathway that had been suggested and the management they had received. There is thus an obligation on the part of caregivers to act in patients' best interests.

\section{Number of visits}

An average of 2000 cataract operations were performed in our unit over the past 3 years. If $90 \%$ of patients are suitable for day case surgery and each patient managed as an SDD saves 1.0 clinic visits, this translates to an annual saving of 1800 clinic visits.

\section{Limitations of the present work}

This study has insufficient power to detect differences in rare complications such as endophthalmitis. A large number of confounder variables are present, but being randomised, the two groups were similar at baseline. The data recorded in clinical practice are unlikely to be as accurate as those obtained in dedicated research clinics. However, this trial has the advantage of reflecting "real world" experience. The researcher was not masked when examining the case notes, but completion of the VCMl questionnaire was exempt from influence of the investigator, and during telephone interviews the researcher was not aware of patient allocation. Treatment of complications was not standardised, but again this represents a real world clinical environment.

\section{CONCLUSION}

The necessity for commencing or changing treatment on day 1 is difficult to quantify for many postoperative conditions. As timing of the development of serious postoperative complications after phacoemulsification surgery cannot accurately be predicted, it is extremely difficult to plan an economically viable review strategy to detect these complications at a presymptomatic stage.

In this trial, the frequency of serious complications detected on the first postoperative day was low. There was no statistically significant difference between the two groups in the proportions achieving a good visual outcome, based on 2 week visual acuity and 4 month quality of life scores. For the overall majority of patients, visual outcome is not compromised when routine next day review is omitted after phacoemulsification surgery.

\section{Authors' affiliations}

C G Tinley, Southampton University Hospitals Trust, Southampton, UK

A Frost, South Devon Healthcare NHS Trust, UK

K N Hakin, W McDermott, P Ewings, Taunton and Somerset NHS Trust, Taunton, UK

\section{REFERENCES}

1 NHS Executive. Action on cataracts. Good practice guidance. London: NHS Executive, February 2000

2 Tan JHY, Newman DK, Klunker C, et al. Phacoemulsification cataract surgery: is routine review necessary on the first post-operative day? Eye 2000; 14:53-5.

3 Allan BDS, Baer RM, Heyworth P, et al. Conventional routine clinical review may not be necessary after uncomplicated phacoemulsification. Br J Ophthalmol 1997:81:548-50.

4 Whitefield L, Crowston J, Little BC. First day follow up for routine phacoemulsification? Br J Ophthalmol 1996;80:148-50.

5 Tufail A, Foss AJE, Hamilton AMP. Is the first day postoperative review necessary after cataract extraction? Br J Ophthalmol 1995;79:646-8.

6 Dinakaran S, Desai SP, Sunder Raj P. Is the first postoperative day review necessary following uncomplicated phacoemulsification surgery? Eye $2000 ; 14: 364-6$.

7 Herbert EN, Gibbons H, Bell J, et al. Complications of phacoemulsification on the first postoperative day: can follow-up be safely changed? J Cataract Refract Surg 1999;25:985-8.

8 Cohen VML, Demetria H, Jordan K, et al. First day post-operative review following uncomplicated phacoemulsification. Eye 1998;12:634-6.

9 Frost NA, Sparrow JM, Durant JS, et al. Development of a questionnaire for measurement of vision-related quality of life. Ophthalmic Epidemiol 1998;5:185-210.

10 Frost NA, Sparrow JM, Hopper CD, et al. Reliability of the VCM1 Questionnaire when administered by post and by telephone. Ophthalmic Epidemiol 2001;8:1-11

11 Desai P, Minassian DC, Reidy A. National cataract surgery survey 1997-8: a report of the results of the clinical outcomes. Br J Ophthalmol 1999;83:1336-40.

12 Desai $\mathbf{P}$, Reidy A, Minassian DC. Profile of patients presenting for cataract surgery in the UK: national data collection. Br J Ophthalmol 1999;83:893-6.

13 Jürgens I, Matheu A, Castilla M. Ocular hypertension after cataract surgery: a comparison of three surgical techniques and two viscoelastics. Ophthalmic Surg Lasers 1997;28:30-6.

14 Bömer TG, Lagrèze WA, Funk J. Intraocular pressure rise after phacoemulsification with posterior chamber lens implantation: effect of prophylactic medication, wound closure and surgeon's experience. Br J Ophthalmol 1995;79:809-13.

15 Barak A, Desatnik H, Ma-Naim T, et al. Early postoperative intraocular pressure pattern in glaucomatous and nonglaucomatous patients. J Cataract Refract Surg 1996:22:607-11.

16 Browning A, Alwitry A, Hamilton R, et al. Role of intraocular pressure measurement on the day of phacoemulsification cataract surgery. J Cataract Refract Surg 2002;28:1601.

17 Rainer G, Menapace R, Findl O, et al. Intraindividual comparison of the effects of a fixed dorzolamide-timolol combination and latanoprost on intraocular pressure after small incision cataract surgery. J Cataract Refract Surg 2001;27:706-10.

18 Lai JS, Chua JK, Leung AT, et al. Latanoprost versus timolol gel to prevent ocular hypertension after phacoemulsification and intraocular lens implantation. J Cataract Refract Surg 2000;26:386-91.

19 Rainer G, Menapace R, Findl O, et al. Randomized fellow eye comparison of the effectiveness of dorzolamide and apraclonidine on intraocular pressure following phacoemulsification cataract surgery. Eye 2000;14:757-60. 